\title{
An Architecture for Situation-Aware Driver Assistance Systems
}

\author{
Matthias Röckl, Patrick Robertson, Korbinian Frank, Thomas Strang \\ Institute of Communications and Navigation, \\ German Aerospace Center, \\ 82234 Wessling/Oberpfaffenhofen, Germany \\ firstname.lastname@dlr.de
}

\begin{abstract}
Current Driver Assistance Systems merely use a minimum set of information. By using additional information of the environment hazardous situations can be detected earlier, more reliably and with a higher precision. This situational information has a significant impact not only on the hazard detection, but also on other modules such as the Human-Machine-Interface or knowledge distribution between vehicles over suitable networks. For a number of reasons situational information is inherently subject to uncertainty. This may be handled by the use of Dynamic Bayesian Networks, which we suggest can be used to represent these dynamic complex systems.

The objective of this paper is to provide an architecture for Driver Assistance Systems that is aware of uncertain situational information in order to prevent accidents and reduce the number of traffic fatalities. It introduces the concepts of Utility-based Knowledge Exchange based on the consideration of other partners' knowledge, the amount of inference that has taken place to identify a situation and current network conditions. Furthermore, we suggest a representation of Hazard Descriptions using Bayesian Network fragments.
\end{abstract}

\section{INTRODUCTION}

Every year about 40,000 people die on European roads [1]. They are fatalities as a result of more than $1.4 \mathrm{M}$ accidents [1]. Countermeasures distinguish between Active and Passive Safety Applications. Passive Safety Applications react on the incidence of an accident or the definite indication of an accident and thus reduce the number of fatalities but not the number of accidents. In contrast to this an Active Safety Application is any application that tries to prevent accidents. Active Safety Applications intervene at the first indication of a potential accident situation (in the remainder also called hazardous situation) and thus act proactively trying to prevent the accident.

In order to prevent accidents one has to understand the reasons that cause accidents. In $95 \%$ of all accidents some human error is involved [2]. Umemura differentiates in [3] between cognitive errors, judgment errors and operation errors. He measured that concerning all accidents $47 \%$ are caused by cognitive mistakes, $40 \%$ by judgment errors and in the remaining $13 \%$ the driver causes the accident by an inappropriate operation. Thus assisting the driver in order to avoid these errors permits the reduction of accident rates.

Systems that assist the driver in her/his task of driving a vehicle are called (Advanced) Driver Assistance Systems [4]. They support the driver's observation, assessment and action. Active Safety Applications of an Advanced Driver Assistance System are for instance the Electronic Stability Control or the Adaptive Cruise Control. According to [4] Adaptive Cruise Control will prevent up to 4000 accidents p.a. in Europe if only $3 \%$ of vehicles are equipped.

Currently an applications such as Adaptive Cruise Control merely use a small subset of situational information and therefore the hazard detection is restricted to a small number of situations. Extending the applications with broader knowledge about their environment (e.g. overall traffic situation, pavement conditions, etc.) will enhance their capability of recognizing hazardous situations earlier, more reliably and with a higher precision. Therefor sensor information of the own vehicle as well as sensor information of other entities (roadside units, vehicles) is required and has to be communicated appropriately.
The objective of this work is to provide an architecture of a Situation-Aware Driver Assistance System (SADAS) that enables situation-aware Active Safety Applications. Section II provides a first insight into the concept of situation awareness. Section III introduces the SADAS and a sample hazard warning application. How uncertainty in the situational information can be handled is discussed in section IV. Finally, the architecture is presented in section V. The paper ends with a summary, conclusion and outlook in section VI.

\section{Situation Awareness}

Driving a vehicle is a complex task: There are numerous influencing parameters that determine the behavior in traffic. To mention only a few, there are a lot of other vehicles (e.g. cars, (motor-)cycles, trucks, buses, etc.) and pedestrians, which share the same road networks, there are complex road courses, bad weather conditions (fog, rain, wind, etc.), different road surface conditions and many more. To always decide correctly, the driver has be aware of all these parameters and their degree of influence in different situations.

A system that assist the driver in her/his task to lead a vehicle over the street network without accidents has to be aware of all these parameters as well.

We call any information that is used by the system to assess a situation a situational information. But not any situational information represents an influencing parameter to any application. If it influences the application the situation information is called relevant for that application. A system that is aware of the relevant situational information is called situation-aware. Usually situational information is measured by sensors. Various sensors are already integrated in a large number of today's vehicles. There are wheel sensors for the Anti-lock Braking System (ABS), rain sensors for the wipers, weight sensors to adapt the braking pressure, GPS sensors for the navigation system, etc. But all these sensors are merely able to detect the situation in the direct vicinity of the own vehicle, whereas the direct vicinity is defined by the operative range of the in-vehicle sensors [5]. Being aware of situational information merely of the 
direct vicinity, implies restrictions on the hazard detection. Extending the situation awareness beyond the direct vicinity facilitates an earlier, more reliably and more precise hazard detection. To achieve this, sensors outside the own vehicle have to be incorporated.

Wireless communication can be used to exchange the situational information measured by distributed sensors between different vehicles or other entities such as roadside units [5]. An important task concerning the interaction is to guarantee a shared understanding of the interacting entities. This concerns the level of signature, semantic, protocol and context as well [6]. If one entity has a different conceptual understanding of the exchanged situational information in only one of these levels, the information is almost valueless.

By being aware of the situational information, hazardous situations can be detected or even predicted at an early stage. The detection of hazardous situations requires an exact description of the hazard and a reasoning process that evaluates situational information and hazard descriptions. Predicting a hazardous situation is a much more complex task. Not all information is predictable and even if it is predictable, it is not guaranteed that the accuracy leads to unambiguous situations.

Speaking about a hazardous situation in a universal understanding is in general not possible. The exact description of the hazardous situation also depends on the driver. A hazard is, for instance, influenced by the driver's attention, the individual driving behavior (risk-averse or risk-loving) or the condition of the own vehicle. So, the hazardous situation has to be adapted by situational information concerning the individual driver and vehicle as well.

The interaction with the driver (Human-Machine-Interface) may also profit from situational awareness. Beside the decision whether an interaction is required considering the needs and capabilities of the driver, situational awareness offers the possibility to determine the type and characteristics of the presentation. Regarding a warning message of a hazardous situation, the message can be presented by a short text fragment in the dashboard or suitably integrated in the head-up display. Optionally it can be enhanced by an acoustical signal according to the current attention of the driver. Or a tactile hint is provided while pushing the turn signal or pressing on the accelerator pedal. Obviously current Driver Assistance System may benefit from the concepts of situation awareness in various areas.

\section{DRIVER Assistance Systems}

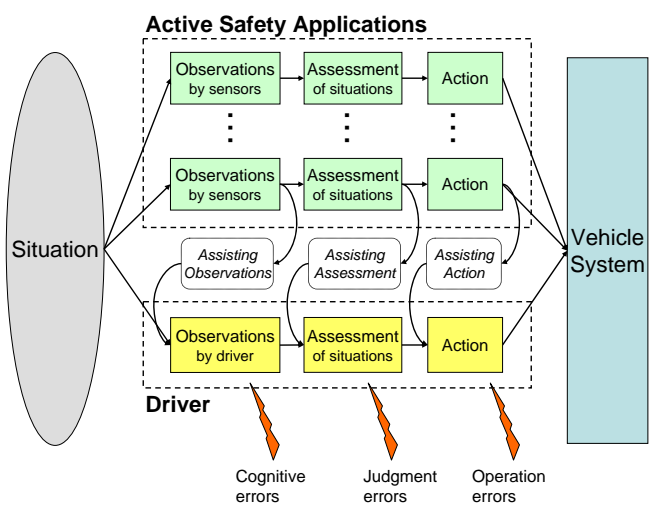

Fig. 1. Support of Driver Assistance Systems

As mentioned in the introduction accidents are mostly based on human cognitive errors, judgment errors and operation errors. These three errors can be associated to three different processes: observation, assessment and action (see fig. 1). An Active Safety Application supports the driver by these processes but it is not mandatory for the application to implement all processes. For instance night-vision applications merely observe the area in front of the vehicle by IR-cameras and assist the driver by providing the observed picture (Assisting Observations). The disadvantage of this type of applications is the information overload for the driver. Usually, it is not reasonable to provide the situational information observed by several types of sensors directly to the driver. This would lead to a huge amount of information which the driver would not be able to process in an adequate time span.

Thus, some applications assess the observed situational information and use the results to initiate some preliminary actions (Assisting Action). A preliminary action would be the preparation of the brake pads if the assessment process detects a short distance to the preceding vehicle and a braking action is anticipated. The subsequent braking action initiated by the driver will be effective earlier. Others such as Adaptive Cruise Control (ACC) implement all three processes and directly interact with the vehicle system. ACC, for instance, measures the distance to the preceding vehicle (observation), recognizes if the distance is too small (assessment) and slows down the vehicle if necessary (action).

Another type of application applies observation and assessment (Assisting Assessment). These applications observe situational information, detect hazardous situations and present the resulting information or warning messages to the driver. Examples for these applications are Traffic Jam Warning, Curve Speed Warning, Road Feature Notification, Intersection Collision Warning, etc. [7]. The remainder of this paper will focus this type of application.

The following section will provide a detailed description of an Active Safety Application.

An everyday situation on the highway is a traffic jam. The vehicles in the traffic jam have a much smaller velocity than the vehicles behind the congestion. So, a common reaction is an abrupt deceleration of the vehicles reaching the end of the jam in order not to collide with the vehicles in front of them. In the worst case the braking action was too late or too diffident and an accident occurs. Probably, this will not be the case if the driver has been informed about the upcoming congestion at an early stage.

In our understanding this warning is not necessarily associated with a traffic jam. It is a warning that informs the driver of one or more slow vehicles in front. The application has to be aware of the velocity of the vehicles driving on the same lane in front of the own vehicle. If they are slow or slow down the application provides a message to the driver to inform her/him about the hazardous situation.

A disadvantage of this approach is the problem that traffic situations are highly dynamic. Vehicles decelerate quickly, but may accelerate quickly as well. That's why a reaction on slow or slowing down vehicles may not satisfy. By using prediction techniques it is possible to anticipate situations in the future and recognize the causes why vehicles will slow down. Exemplary causes why vehicles decrease their speed are bad weather conditions, bad pavement surface, road constriction, slow vehicles in front, red traffic lights, etc. On multi-lane roads it may happen that slow vehicles may change on the driver's lane, because of an ending lane, slow vehicles in front, obstacles on the lane, etc.

In all these cases a Situation-Aware Driver Assistance System may warn or inform the driver about the hazardous situation and thus accidents may be prevented or at least mitigated.

\section{UNCERTAINTY}

A key accessor to Situation Awareness is a well defined model for situational information [14]. Here one has to take into account that not every situational information can be expressed by certain values. Some situational information will remain uncertain. Uncertainty emerges for instance due to:

- Noise in sensor data

- Insufficient temporal or spacial sensor readings

- Malfunction of sensors

- Unreliable wireless data exchange

- Manipulation by malicious intruders 
- Unpredictable behavior

This uncertainty has to be dealt with. In an uncertain environment the optimal solution is to explicitly represent this uncertainty and to take it into account in all forms of subsequent processing. Hence, the knowledge of uncertainty is much better than false assumptions of reliability. In the case, for example, where one is very unsure if the vehicle in front has slowed abruptly, it might be premature to perform a braking maneuver oneself but advantageous to apply the brake pads in preparation.

One approach to deal with uncertainty in complex systems is the usage of Bayesian Networks. A Bayesian Network is a probabilistic graphical model comprising a set of nodes which represent random variables and directed edges representing conditional dependencies. Every node has an associated conditional probability distribution (or prior distribution if there are no parent nodes) specified in a Conditional Probability Table (CPT) if the node represents a discrete random variable or a conditional Probability Density Function (PDF) if the random variable is continuous.

In Bayesian Networks the directed relations are often interpreted as causal dependencies, whereby the strength of causal relationships is encoded in the CPT or PDF [8]. A conditional probability associated with an edge from $A$ to $B$ can be regarded as the probability that $A$ causes $B$. Thus, the probability of $B$ given its Markovian parents $A_{1}, \ldots, A_{n}$ is the conditional probability $P\left(B \mid A_{1}, \ldots, A_{n}\right)$ [9]. Regarding a traffic situation a causal relation might be expressed as follows: If the vehicle in front is following a slow truck, this might result in a lane changing manoeuvre of this vehicle.

But it is also possible to apply the Bayesian Network from a diagnostic point of view. The objective is to reason from a given effect $B$ to the likelihood of any of its possible causes $A$. If for example, the wheel sensors detect poor slip and the temperature is below zero, the slip might be the result of icy road conditions.

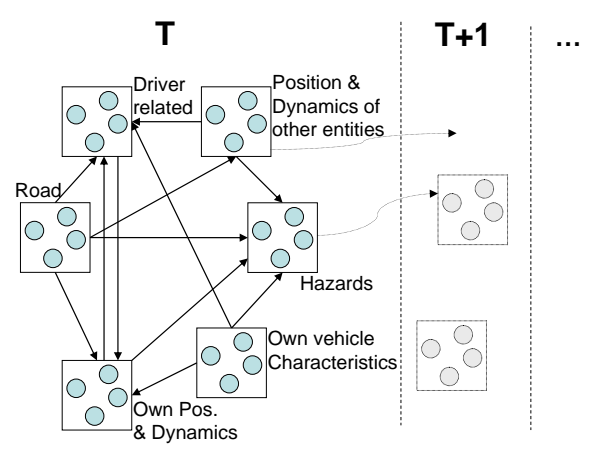

Fig. 2. Dynamic Bayesian Network

A special form of Bayesian Networks is the dynamic Bayesian Network considering time-discrete stochastic processes [10]. Dynamic Bayesian Networks are used for representing complex dynamic systems. They consist of collections of random variables partitioned into input, hidden and output variables. These random variables are separated in different time slices (see fig. 2). Modeling, for example, driver behavior with dynamic Bayesian Networks enables the prediction of future states to enhance the detection of hazardous situations. It has to be mentioned that the future states represent merely a belief that this state will be reached. That is why Bayesian Networks are also called Belief Networks.

\section{Architecture}

After showing the benefit and peculiarities of using situation awareness in Driver Assistance Systems we will now present an architecture for the Situation-aware Driver Assistance System. The main parts of our architecture are the Utility-based Knowledge Exchange component, the Knowledge Broker, the Reasoner and the HumanMachine-Interface/Machine-Machine-Interface (HMI/MMI) (see fig. 3).

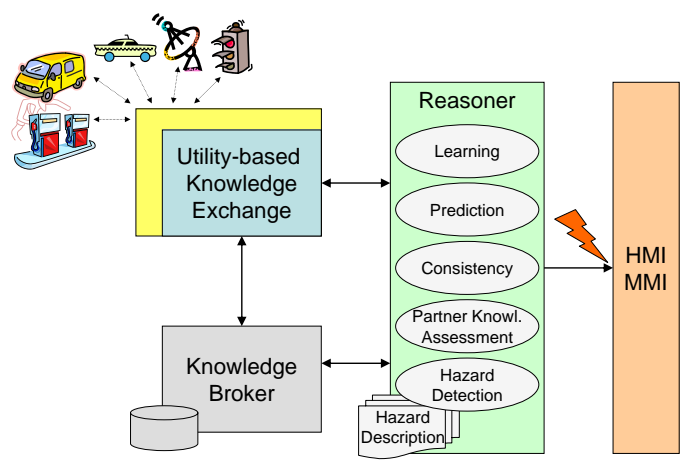

Fig. 3. SADAS core components

\section{A. Utility-based Knowledge Exchange}

The Utility-based Knowledge Exchange component is responsible for the interaction with other vehicles (inter-vehicle communication), road-side units or any other entity. It receives situational information from these entities and distributes own situational information to other entities.

Among other important tasks the Utility-based Knowledge Exchange component is responsible for the selection of a suitable network, the consideration of knowledge of the network partners and the trade-off concerning the level of knowledge inference (see fig. 4. These tasks are strongly interdependent and collaborate closely. The following paragraphs discuss these three tasks in detail.

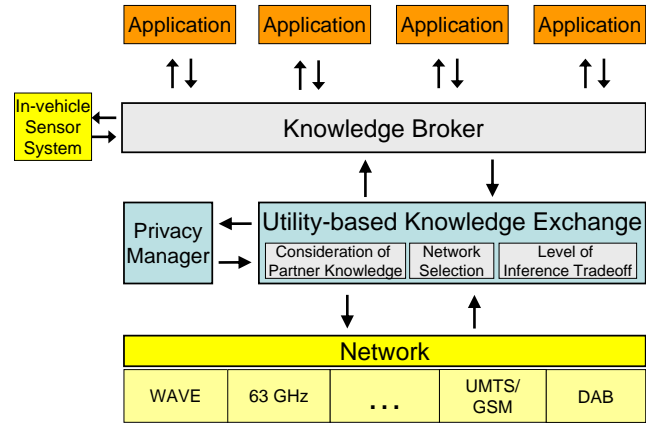

Fig. 4. Knowledge exchange in SADAS

a) Network selection: The exchange of situational information can be based on different wireless network technologies mainly differentiated by ad-hoc and infrastructure setup. Ad-hoc networks are for instance WLAN (specified by IEEE $802.11 \mathrm{a} / \mathrm{b} / \mathrm{g} / \mathrm{p}$ ), InfraRed (IR) or millimeter wave (63 GHz). GSM/UMTS, Radio Data System (RDS), Digital Audio Broadcast (DAB) are examples of infrastructure networks. The networks are characterizable by their data rate, their delay, their reliability, their range, their monetary costs, etc. Here, these characteristics do not represent the potential values (e.g. max. data rate) but the actual values (e.g. the actual available data rate). Every network can be characterized by these values. When information has to be exchanged requirements specify the expected 
characteristics of the interaction (e.g. max. delay). The requirements depend on the type of information, the urgency, etc. The complex task of selecting the best suitable network according to the specified requirements is done by the network selection process.

In Continuous Air interfaces - Long and Medium range (CALM) [11] the selection of the best suitable network is done by matching the requirements of the exchanging information against the characteristics of the network. This is not taking into account the situational conditions (such as volume of traffic). But situational conditions may also play a crucial role in the network selection. Distributing information by a long-range communication network while being in a traffic jam encourages network overload. On the other hand if there is a low traffic density, information has to be distributed by long-range communication in order to reach other vehicles. Therefore the network selection in our architecture takes into account relevant situational information and uses the optimal network for the knowledge exchange.

b) Consideration of partner knowledge: The idea behind the knowledge exchange is the objective that every entity is aware of all relevant situational information. If an entity lacks some situational information or merely is aware of outdated situational information it may request it. But to achieve this, the entity has to know that some relevant situational information is missing or outdated. This is often not the case.

On the other hand the holder of the knowledge may initiate the knowledge exchange. Every time the holder gets new information it disseminates it to its network partners. This would lead to a network overload in the majority of cases because of the great number of sensors that provide numerous updates in highly dynamic environments. Countermeasures to reduce the number of exchanged information are, for instance, the implementation of threshold- and/or hysteresis-based approaches or periodic updates.

Another approach originated from cooperative robot systems uses an estimation of the partner's knowledge to assess which information the partner requires. That means that an entity should send the situational information that increases the knowledge of the partner in the best way. This can also be used for prioritising the knowledge exchange. If, for example, a vehicle enters the highway it may not be aware of the situational information concerning the highway (e.g. max. speed, traffic situation on the highway). By knowing the position of the vehicle on the highway approach, an information holder prioritises and disseminates the relevant situational information to this vehicle. Accordingly, important situational information (e.g. position of an approaching vehicle with high velocity) is disseminated first.

This task also considers aspects concerning grouping or individualization of knowledge exchange. If there are entities which have an equal standard of knowledge the situational information exchange may be grouped to these entities. In some cases it may also be reasonable to exchange a reduced number of situational information because multiple partners require an identical set of situational information. Then, the situational information can be grouped to a common set and disseminated to these entities. Additional information may be exchanged individually.

This also impacts the network selection task in choosing a suitable network for the grouped/individual information exchange. For example, the exchange of situational information adapted to an individual entity may utilize a dedicated unicast network; the exchange of grouped situational information is done by multicast, broadcast or geocast [12]

c) Level of knowledge inference tradeoff: A third task of the Utility-based Knowledge Exchange component is the assessment of the knowledge inference level.

Situational information can be described in different levels of inference. The lowest level is raw sensor data. On the contrary, a concrete warning message is highly inferred. Exchanging situational information by its raw sensor data leads to a broad knowledge enhancement of the partner but consumes a lot of bandwidth. On the other hand, highly inferred situational information consists of less detailed information but therefore consumes less bandwidth.

Transmitting highly inferred information causes problems if the inferred information is incorrect but the receiver assumes that it is correct and distributes it to other entities. This would quickly escalate to a situation in which incorrect knowledge is distributed. Other entities are not able to verify it because of the lack of the underlying detailed information [13].

This task also collaborates with the other tasks. It takes into account the network characteristics in order to adapt the level of knowledge inference. If the partner requires some situational information within a short delay but the network is affected by overload it selects highly inferred situational information. This information consumes less bandwidth and comprises the information urgently required by the partner.

\section{B. Knowledge Broker}

The Knowledge Broker is responsible for storing and retrieving of situational information. The Knowledge Broker does not differentiate between situational information originated from other vehicles, the own vehicle, or any other entity. Situational information generated by in-vehicle sensors may be represented by wrapper data sets which retrieve the sensor data directly when needed.

\section{Reasoner}

The Reasoner is a central component of our architecture. The process of reasoning has to deal with the uncertainty of the situational information. Reasoning in general stands for the process of deriving concluding expressions or facts from a set of other expressions or facts based on a logic.

We will use reasoning in our system mainly for the following tasks:

a) Learning: The objective of learning is the definition or update of the structure (random variables and conditional dependencies) and the conditional probability distributions (CPDs) of the model. Usually, learning is based on inductive reasoning such as Bayesian learning. Bayesian learning derives CPDs from observations and prior knowledge. Prior knowledge is given by domain experts, and observations (i.e. training data) may be acquired during runtime.

For instance, learning can help to characterize driving behavior. Result may be a characterization of the driving behavior such as an instance of careful or risky driver. This can be used, for example by the Slow Vehicle Warning application, to estimate whether the driver will change the lane suddenly because of a slow vehicle in front or will stay behind the slow vehicle.

b) Hazard Detection: Hazard Detection is a central function of the Reasoner and essential for the functionality of the whole system. The overall objective is to detect hazardous situations at an early stage.

There are different types of hazardous situations. A hazardous situation may result in an accident. This accident may be caused by a collision with another vehicle. A collision is an unfavorable constellation of the involved vehicles. The constellation is influenced by the position and the velocity of the own and other vehicles.

The above described dependencies can be represented by a Bayesian Network fragment [15] (see fig. 5). This fragment is the basis for the hazard detection. Different types of hazardous situations are specified by appropriate Bayesian Network fragments. To extend the system to detect further hazardous situations new Bayesian Network fragments can be integrated into the system. These fragments are dynamically loaded.

The logical background of the hazard detection is plausible reasoning [16]. Deductive reasoning is not suitable because of the uncertainty of the premises. By using plausible reasoning it is possible to draw conclusions from uncertain priors and evidence. The conclusions are therefore plausible stated by a probability. For example, given the vehicle in front might slow down due to evidences in the traffic situation and learned driving behavior, a hazardous situation is supposed to occur and the driver has to be warned. The 


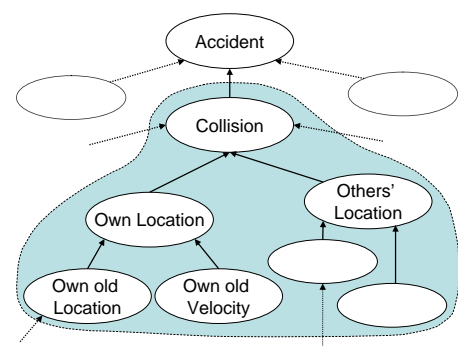

Fig. 5. Bayesian Network Fragment

probability of the accident occurrence is handled appropriately in the HMI/HMM.

c) Prediction: According to [17] prediction is the computation of the probability distribution for all possible situational information for a future point in time. Prediction may be based on plausible reasoning as well because of the uncertain premises. Using prediction to anticipate future states offers a much broader scope to the hazard detection. Hazardous situations may be detected at an early stage and thus, the driver may be warned in time.

d) Assessment of partner's knowledge: The Utility-based Knowledge Exchange component considers the knowledge of network partners to optimize the exchange of situational information (see sec. V-A). The assessment of the partner's knowledge is part of the reasoning process. The Reasoner provides this information to the Utility-based Knowledge Exchange component.

Furthermore, the hazard detection and the triggering of appropriate actions are based on the assessment of the partner's knowledge as well. Being aware of the knowledge of other traffic participants positively contributes to the prediction of the behavior in the future. For example, if the assessment of the partner's knowledge notices that the partner is not aware of the own position because the own vehicle is in the blind spot of the partner, a warning message may be provided to the driver.

e) Consistency Check: Another task of the Reasoner is the checking for consistency of the situational information. The consistency check may detect manipulations by malicious intruders or the malfunction of sensors that provide incorrect data to the system. This information may then be neglected or becomes less relevant in other reasoning tasks.

\section{D. $H M I / M M I$}

The Human Machine Interface or Machine Machine Interface (HMI/MMI) is triggered by the system if a hazardous situation is detected. It expresses the hazard in a proper way. This may be achieved by visual (text, depiction, etc.), verbal, tactile (e.g. vibrating accelerator pedal) signals or any other type of warning notices. Depending on situational information and the hazard type a visual notice may be depicted on the dashboard, the head-up display, etc. The type of depiction is dependent on the urgency, the probability, the type of hazard, etc.

\section{Summary, Conclusions And Outlook}

This paper has identified major architectural components of a Situation-Aware Driver Assistance System. We focussed on Driver Assistance Systems that use sensor based knowledge derivation and which rely on inter-vehicle and infrastructure-to-vehicle network connectivity. We have placed these safety applications into three main domains namely observation, assessment and action depending on the stage at which they can positively support the driver, in the chain from observing the current situation to reacting appropriately. We focus on applications that support the correct assessment of the situation, letting the driver perform the correct task; nevertheless, the principles and suggested architecture will support applications that support actions themselves or even perform these to prevent an accident.

We propose an architecture for a complete system that supports driver assistance applications and describe the main components: 1) a Knowledge Broker that stores and retrieves situational information, 2) a Utility-based Knowledge Exchange that optimizes the exchange of situational information between different entities, 3) a Reasoner that supports learning of the underlying model, prediction of future states and hazard detection with dynamic loading of hazard descriptions.

A focus of our work was on the employment of (dynamic) Bayesian Networks to represent and infer the relevant knowledge given the observation of available sensors and information about the other vehicles, etc. In particular, we suggest that such a network can be used to generate probabilities of hazards, which can then be used to govern the appropriate interaction with the user. This approach also allows us to make decisions on which knowledge should be distributed to partner vehicles in our vicinity, and easily allows dynamic incorporation of hazard descriptions in the form of network fragments.

Our next steps are to analyse the performance of the SituationAware Driver Assistance System in a simulation of communications network behavior and real traffic situations.

\section{REFERENCES}

[1] European Commission - Transport. CARE - Community Road Accident Database. http://ec.europa.eu/transport/roadsafety/ road_safety_observatory/care_en.htm.

[2] Andre Vits. Workshop on sprectrum requirements. Technical report, European Commission, Directorate General Information Society and Media, 2006.

[3] Yoshiyuki Umemura. Driver Behavior and Active Safety. In $R \& D$ Review of Toyota CRDL - Special Issue : Driver Behavior and Active Safety, 2004.

[4] Johannes Abele et al. Exploratory study on the potential socio-economic impact of the introduction of intelligent safety systems in road vehicles. Technical report, VDI/VDE Innovation - Technik GmbH \& Institute for Transport Economics at the University Cologne, 2005.

[5] Luisa Andreone and Michele Provera. Inter-vehicle communication and cooperative systems: local dynamic safety information distributed among the infrastructure and the vehicles as "virtual sensors" to enhance road safety.

[6] Thomas Strang and Claudia Linnhoff-Popien. Service interoperability on context level in ubiquitous computing environments. In International Conference on Advances in Infrastructure for Electronic Business, Education, Science, Medicine, and Mobile Technologies on the Internet, January 2003.

[7] Car-2-Car Communication Consortium. http://www.car-2-car.org.

[8] David Heckerman. A tutorial on learning with bayesian networks. Technical report, Microsoft Research Advanced Technology Division - Microsoft Corporation, 1995.

[9] Judea Pearl. Causality: Models, Reasoning, and Inference. Cambridge University Press, 2000.

[10] Kevin Patrick Murphy. Dynamic Bayesian Networs: Representation, Inference and Learning. PhD thesis, 2002.

[11] Continuous Air interfaces - Long and Medium range (CALM) - ISO 21210.

[12] Timo Kosch. Situationsadaptive Kommunikation in Automobilen Ad-hoc Netzen. PhD thesis, Technical University of Munich, 2005.

[13] BMW Private communications with Markus Strassberger.

[14] Thomas Strang and Claudia Linnhoff-Popien. A context modeling survey. In First International Workshop on Advanced Context Modelling, Reasoning And Management at UbiComp 2004, Nottingham, England, September 7, 2004, September 2004.

[15] Kathryn Blackmond Laskey and Suzanne M. Mahoney. Network fragments: Representing knowledge for constructing probabilistic models. In Thirteenth Conference on Uncertainty in Artificial Intelligence. Morgan Kaufmann Publishers, 1997.

[16] E.T. Jaynes. Probability Theory: The Logic of Science. Cambridge University Press, 2003.

[17] Rene Michael Mayrhofer. An architecture for Context Prediction. PhD thesis, 2004. 